\title{
Análisis de la relación amplitud-frecuencia en las señales EEG mediante Redes Bayesianas
}

\author{
Gabriela García Manzo ${ }^{1,3}$, Guillermo De la Torre-Gea ${ }^{2}$ \\ ${ }^{1}$ Instituto Nacional de Neurociencias, Psicoanálisis y Salud Mental, Av. México No. Col. \\ Hipódromo Condesa, Del. Cuauhtémoc, D.F., México. \\ ${ }^{2}$ CA Biosistemas. Universidad Tecnológica de Corregidora, Carretera Estatal 413 \\ Querétaro-Coroneo km 11.2 Qro. Tel. (442)4830070. \\ ${ }^{3}$ Universidad Politécnica de Santa Rosa Jáuregui, Carretera Querétaro-San Luis Potosí, km \\ 31+150, Parque Industrial Querétaro Sta. Rosa Jáuregui, Qro. Tel. (442)2409520.
}

\begin{abstract}
Resumen: En este trabajo, se propone un método para Interfaces CerebroComputadora basado en modelos gráficos probabilísticos que supera algunas de las limitaciones de los enfoques basados en clasificadores de señales. Se modela la dinámica del cerebro a nivel de las relaciones amplitud-frecuencia ocultas, utilizando una red bayesiana dinámica que se aprende a partir de datos obtenidos de Electro-encefalogramas. Se demuestra cómo un el modelo puede utilizarse para inferir las distribuciones de probabilidad sobre variables de estado ocultas, las cuales corresponden a funciones cerebrales útiles para establecer un modelo de de interface. El uso del modelo nos proporcionó una estimación probabilística continua de estado del cerebro, permitiendo modelar la estructura causal y dependencias entre los diferentes estados cerebrales. Este modelo gráfico dinámico en el tiempo permite realizar estimaciones aún con datos faltantes, y el suavizado de las estimaciones de estado, utilizando la información de los futuros puntos de datos. Estas capacidades son difíciles de obtener, al trabajar exclusivamente en el dominio de frecuencias.
\end{abstract}

Palabras clave: Cerebro, Redes Bayesianas, BCI, EEG.

\section{Introducción}

Las redes neuronales distribuidas aseguran el correcto funcionamiento del cerebro humano (Mesulam, 1998). Los procesos de alimentación directa y retroalimentación inhibitorios y excitatorios son los mecanismos básicos de la interacción entre los diferentes módulos de estas redes (Bullier, 2001). La localización de los diferentes módulos de la red funcional, implicando una tarea mental dada, es el objetivo principal de los estudios de Interfaces Cerebro-Computadora (BCI por sus siglas en inglés). Una gran cantidad de investigaciones, usando Tomografía de Emisión de Positrones y la Resonancia Magnética Funcional, se han dedicado a este objetivo (Cabeza y Nyberg, 2000). Sin embargo, estos métodos no son los más adecuados para determinar cuándo los diferentes módulos se vuelven activos durante la tarea mental y, por tanto, en qué etapa de procesamiento está involucrado cada módulo. Tampoco pueden fácilmente responder a las preguntas importantes de la activación 
secuencial de alimentación, en paralelo a los procesos de retroalimentación, o cómo la información está "obligada" para formar percepciones unificadas.

Con el fin de investigar tales propiedades temporales de los circuitos del cerebro, es necesario utilizar métodos que midan directamente la actividad neuronal en tiempo real. El Electro-encefalograma (EEG) ofrece esta posibilidad mediante la medición de la actividad eléctrica de los cúmulos de células neuronales en una escala temporal de micro segundos. Desafortunadamente, estas técnicas se enfrentan al problema de que las señales medidas en la superficie del cuero cabelludo no indican directamente la ubicación de las neuronas activas en el cerebro debido a la ambigüedad del problema subyacente de la estática electromagnética inversa.

El EEG mide la actividad eléctrica cerebral causada por el flujo de corrientes eléctricas durante excitaciones sinápticas de las dendritas en las neuronas y es extremadamente sensible a los efectos de las corrientes secundarias (Baillet et al., 2001). Las señales de EEG se registran fácilmente de una manera no invasiva a través de electrodos colocados en el cuero cabelludo, lo cual corresponde a la modalidad de grabación más generalizada. Sin embargo, proporciona señales de muy mala calidad, ya que las señales deben cruzar el cuero cabelludo, el cráneo y otras capas. Esto significa que las señales de EEG en los electrodos son débiles, difícil de adquirir y de mala calidad. Además, esta técnica es gravemente afectada por el ruido de fondo generado tanto en el interior del cerebro o externamente sobre el cuero cabelludo.

Un EEG comprende un conjunto de señales que se pueden clasificar de acuerdo a su frecuencia. Los rangos de frecuencia más conocidos se han definido de acuerdo con la distribución a lo largo del cuero cabelludo, de importancia biológica. Estas bandas de frecuencia se denominan como Delta $(\delta)$, Theta $(\theta)$, Alfa $(\alpha)$, Beta $(\beta)$, y $\operatorname{Gamma}(\gamma)$.

La amplitud de las bio-señales eléctricas está en el orden de micro voltios $(\mu \mathrm{V})$. En consecuencia, la señal es muy sensible a ruido electrónico. Fuentes externas y líneas eléctricas pueden generar ruido de fondo, como la sensación térmica, movimientos, parpadeo, y los ruidos generan ráfagas en las señales internas (Leach, 1994). Las consideraciones al estudiar estas señales para el desarrollo de BCI deben dirigirse a reducir los efectos del ruido, tales como el blindaje electromagnético de interferencia o la reducción de la señal de forma manual, entre otros (Usakli, 2010).

La determinación de patrones de comportamiento en la señal que comunica a las estructuras del cerebro es difícil de realizar, debido a la complejidad de su anatomía y funcionamiento, por lo que es necesario incorporar nuevas técnicas que tomen en cuenta muchas variables a la vez. El objetivo de este estudio es abordar este problema mediante el enfoque de las Redes Bayesianas (BN por sus siglas en inglés), para describir las relaciones entre las frecuencias estudiadas como variables. Las BN son técnicas numéricas de incertidumbre que hacen uso de la inferencia bayesiana como método heurístico (De la torre- Gea et al., 2011a). 


\section{Teoría de redes bayesianas}

Las $\mathrm{BN}$ son tipos de representación del conocimiento desarrollado en el campo de la inteligencia artificial para realizar aproximaciones en el campo del razonamiento (Pearl, 1988; Mediero, 2007; Gámez et al, 2011; Zaidan et al, 2011). Una BN es un gráfico acíclico cuyos nodos corresponden directamente a los conceptos o variables aleatorias y cuyos enlaces se corresponden con las relaciones o funciones (Correa et al., 2009). Las variables se definen en un dominio discreto o cualitativo, y las relaciones funcionales describen las inferencias causales expresadas en términos de probabilidades condicionales que se muestra en la ecuación (1):

$$
\mathrm{P}(\mathrm{x} 1, \ldots, \mathrm{xn})=\prod_{i=1}^{n} \mathrm{P}(\mathrm{xi} \mid \text { parents }(\mathrm{xi}))
$$

Una $\mathrm{BN}$ se puede utilizar para identificar las relaciones entre las variables anteriormente indeterminadas o para describir y cuantificar estas relaciones, incluso con un conjunto de datos incompletos (Hruschka et al, 2007; Reyes, 2010). El algoritmo de solución de $\mathrm{BN}$ permite el cálculo de la distribución de probabilidad esperada de las variables de salida. El resultado de este cálculo depende de las distribuciones de las probabilidades de las variables de entrada. A nivel global, las BN pueden ser percibidas como una distribución de probabilidad conjunta de una colección de variables aleatorias discretas (Garrote et al., 2007).

$$
P(c j \mid x i)=P(x i \mid c j) P(c j) / \sum_{k} P(x i \mid c k) P(c k)
$$

Una probabilidad a priori $\mathrm{P}(c j)$ es una probabilidad de que una muestra $x i$ pertenezca a la clase $c j$, sin ninguna información sobre sus valores característicos, como se muestra en la ecuación (2). Las máquinas de aprendizaje en inteligencia artificial, están relacionadas con los métodos de minería de datos, la clasificación o agrupación y el reconocimiento de patrones. Los métodos estadísticos de aprendizaje automático se pueden aplicar al marco de la estadística bayesiana, sin embargo el aprendizaje automático se puede emplear en una variedad de técnicas de clasificación para producir otros modelos de BN. El objetivo de una BN de aprendizaje es encontrar un arreglo de red que mejor describa los datos observados.

En los modelos de aprendizaje, el método más representativo es el de "búsqueda y resultado" basado en el algoritmo K2. Dicho algoritmo comienza asignado un nombre a cada variable sin "padres". A continuación agrega a cada variable un padre de forma incremental, la cual en su mayoría aumenta la puntuación de la estructura resultante. Cuando cualquier adición no puede aumentar el marcador, deja de aumentar padres a la variable. Tomando en cuenta un conocimiento previo del ordenamiento de las variables con base a su grado de dependencia, el espacio de búsqueda en esta restricción es mucho menor que el espacio de toda una estructura y no hay necesidad de compro- 
bar los ciclos en el proceso de aprendizaje. Si no se tiene conocimiento anterior sobre el ordenamiento de las variables, se procede a nuevas búsquedas (Guoliang, 2009).

\section{Materiales y métodos}

Para desarrollar éste trabajo, se empleó una computadora con procesador Intel core-i7 con 8 GB de memoria RAM y sistema operativo Windows 8. Los datos fueron discretizados mediante el sistema ELVIRA, como se muestra posteriormente para ser empleados en el desarrollo del modelo de Redes Bayesianas, que describe las relaciones entre todas las variables.

Se obtuvieron 20 conjuntos de datos a partir de EEGs aplicados a distintas personas, bajo las mismas condiciones basales, en el Centro Estatal de Salud Mental (CESAM) del Estado de Querétaro, quien proporcionó dichos datos mediante archivos en formato de texto plano (txt). Cada archivo fue convertido a formato de texto delimitado por espacios (csv) mediante el programa MS Excel y organizado en 48 columnas, cada una correspondiente a una frecuencia de las señales EEG en un rango de 0.781 a $19.144 \mathrm{~Hz}$, las cuales fueron definidas como las variables aleatorias. Cada columna contó con 3564 datos de voltaje medido en $\mu \mathrm{V}$, que corresponden a las fuentes de las señales EEG, provenientes de diferentes estructuras del cerebro.

Cada fuente de señal EEG expresa un valor de amplitud en $\mu \mathrm{V}$ para un valor de frecuencia dado. Por lo que cada nodo de la BN se encuentra dentro de una frecuencia cerebral. De esta manera, la BN muestra las relaciones de dependencia entre frecuencias de las señales cerebrales con base al comportamiento de sus voltajes.

El análisis de BN fue realizado mediante el software ELVIRA versión 0.162 en tres etapas sugeridas por Mediero (2007).

a) Pre-procesamiento: Se llevó a cabo mediante el algoritmo de imputación "por promedios" para completar las series de datos parciales. Este algoritmo reemplaza los valores faltantes o desconocidos, por el promedio de los valores para cada variable. Este método no necesita parámetros y consiste en la discretización de los datos masivos mediante el algoritmo, empleando seis intervalos con la misma frecuencia.

b) Procesamiento: De acuerdo con Wang et al. (2006), la mejor estructura de red bayesiana se obtiene empleando el algoritmo K2 con un número máximo de padres igual a cinco y sin restricciones.

c) Post-procesamiento: Se realizó un análisis de dependencias para obtener la estructura topológica de la red, la cual representa a las variables y sus dependencias causales. Después de obtener la red de aprendizaje paramétrico, se realizó el cálculo de las probabilidades condicionales en las variables que muestren relación o dependencia. 


\section{Resultados y discusión}

Se obtuvo un modelo de BN con un $97.5 \%$ de precisión, calculado en la etapa de post-aprendizaje mediante el software ELVIRA, el cual muestra las relaciones entre las variables estudiadas. La Tabla 1 detalla un resumen del modelo de $\mathrm{BN}$ obtenido a partir de los 20 conjuntos de datos, el cual expresa las relaciones de dependencias entre las frecuencias, a partir de sus valores de voltaje.

Tabla 1. Resumen de los resultados obtenidos mediante BN.

\begin{tabular}{|c|c|c|c|c|}
\hline Id & Frecuencia $(\mathrm{Hz})$ & Dependencias & Probabilidad condicional & Amplitud $(\mu \mathrm{V})$ \\
\hline 1 & 0.781 & 2 & 0.165263749 & 3.69 \\
\hline 2 & 1.172 & 3,4 & 0.930390492 & 3.32 \\
\hline 3 & 1.563 & 4,5 & 0.972927242 & 2.95 \\
\hline 4 & 1.953 & 5 & 0.984347826 & 2.76 \\
\hline 5 & 2.344 & $6,36,48$ & 0.989637306 & 2.61 \\
\hline 6 & 2.7347 & 7 & 0.979695431 & 2.42 \\
\hline 7 & 3.1254 & $8,9,33,35$ & 0.967632027 & 2.39 \\
\hline 8 & 3.5161 & 9,10 & 0.983164983 & 2.2 \\
\hline 9 & 3.9068 & 10,11 & 0.988013699 & 2.13 \\
\hline 10 & 4.2975 & $11,12,13$ & 0.979345955 & 2.03 \\
\hline 11 & 4.6882 & $12,31,32$ & 0.994764398 & 1.9 \\
\hline 12 & 5.0789 & 13,15 & 1 & 1.81 \\
\hline 13 & 5.4696 & $14,15,16$ & 0.965576592 & 1.82 \\
\hline 14 & 5.8603 & $16,17,34$ & 0.97972973 & 1.66 \\
\hline 15 & 6.251 & 18,30 & 1 & 1.56 \\
\hline 16 & 6.6417 & $17,19,20$ & 0.936206897 & 1.63 \\
\hline 17 & 7.0324 & $18,20,22$ & 0.998165138 & 1.42 \\
\hline 18 & 7.4231 & 19 & 0.989399293 & 1.27 \\
\hline 19 & 7.8138 & $21,22,24$ & 0.98568873 & 1.32 \\
\hline 20 & 8.2045 & 21 & 0.98943662 & 1.16 \\
\hline 21 & 8.5952 & 18,19 & 0.987719298 & 1.18 \\
\hline 22 & 8.9859 & 23,28 & 0.987632509 & 1.19 \\
\hline 23 & 9.3766 & 24,25 & 0.992945326 & 1.16 \\
\hline 24 & 9.7673 & 25,26 & 0.968911917 & 1.21 \\
\hline 25 & 10.158 & 26,27 & 0.994708995 & 1.31 \\
\hline 26 & 10.5487 & 27 & 0.975986278 & 1.38 \\
\hline 27 & 10.9394 & 28 & 0.994809689 & 1.54 \\
\hline 28 & 11.3301 & 29 & 0.988950276 & 1.6 \\
\hline 29 & 11.7208 & 30 & 0.962774958 & 1.59 \\
\hline 30 & 12.1115 & 31 & 0.992307692 & 1.45 \\
\hline 31 & 12.5022 & 32 & 0.996138996 & 1.39 \\
\hline 32 & 12.8929 & 33 & 0.992409867 & 1.06 \\
\hline 33 & 13.2836 & 34 & 0.992366412 & 0.82 \\
\hline 34 & 13.6743 & 35 & 0.994594595 & 0.64 \\
\hline 35 & 14.065 & 36 & 0.998161765 & 0.52 \\
\hline 36 & 14.4557 & 37,38 & 0.983082707 & 0.42 \\
\hline 37 & 14.8464 & 38,39 & 0.974619289 & 0.33 \\
\hline 38 & 15.2371 & $39,40,41$ & 0.987847222 & 0.33 \\
\hline 39 & 15.6278 & 40 & 0.987826087 & 0.33 \\
\hline 40 & 16.0185 & $41,42,43,46,47$ & 0.984320557 & 0.3 \\
\hline 41 & 16.4092 & 42 & 0.989583333 & 0.28 \\
\hline
\end{tabular}


Gabriela García y Guillermo De la Torre

\begin{tabular}{lcccc}
\hline 42 & 16.7999 & $43,44,45$ & 0.991304348 & 0.29 \\
43 & 17.1906 & 44 & 0.994773519 & 0.26 \\
44 & 17.5813 & 45 & 0.996515679 & 0.25 \\
45 & 17.972 & 45,47 & 0.987993139 & 0.25 \\
46 & 18.3627 & 48 & 0.991334489 & 0.23 \\
47 & 18.7534 & 39,44 & 0.996533795 & 0.23 \\
48 & 19.1441 & & 0.998154982 & 0.12 \\
\hline
\end{tabular}

La BN obtenida muestra patrones de dependencias entre nodos dentro de los intervalos de frecuencias Alfa, Beta, Delta y Theta que sugieren similitud en el comportamiento de los valores de voltaje para cada intervalo, no de los valores en sí; y entre nodos de diferentes intervalos, en menor número, que muestran dependencia entre frecuencias.

La banda Delta se encuentra por debajo de $4 \mathrm{~Hz}$, y la amplitud de las señales Delta disminuye a medida que se envejece. Los ritmos Delta en general sólo se observan en adultos en estado de sueño profundo y son poco frecuentes en adultos en un estado despierto (Kubler et al., 2001). En este intervalo se observan pocas dependencias entre nodos.

Las ondas Theta se encuentran dentro de la gama de 4 a $7 \mathrm{~Hz}$. En un adulto despierto normal sólo una pequeña cantidad de frecuencias Theta se puede grabar, ya que se encuentran en adultos soñolientos, meditación o sueño ligero (Kubler et al., 2001). Las frecuencias Theta han sido asociadas con la concentración meditativa (Anand et al., 1961; Aftanas y Golocheikine, 2001) y una amplia gama de procesos cognitivos como el cálculo mental (Fernández et al., 1995), demandas de la tarea en laberintos (Caplan et al., 2001), o la conciencia (Klimesch et al., 2001). Se observa una mayor cantidad de dependencias de nodos del mismo intervalo de frecuencia y con nodos de la frecuencia Beta.

Los ritmos Alfa se encuentran sobre la región occipital en el cerebro (Jaime, 2005). Estas ondas se observan dentro del intervalo de 8 a $12 \mathrm{~Hz}$. Su amplitud aumenta cuando los ojos se cierran y el cuerpo se relaja y se atenúa cuando se hace el esfuerzo mental y los ojos abiertos (Black, 1972). Estos ritmos reflejan principalmente el procesamiento visual en la región occipital del cerebro y también pueden estar relacionados con la función de memoria (Klimesch, 1997). También hay pruebas de que la actividad Alfa puede estar asociada con un esfuerzo mental, sin embargo, generalmente causa su supresión, en particular de las áreas frontales (Venables y Fairclough, 2009). En consecuencia, estos ritmos pueden ser señales útiles para medir el esfuerzo mental (Pfurtscheller et al., 2006). En este intervalo se observan pocas dependencias entre nodos y ninguna con nodos de otras frecuencias.

Las frecuencias Beta, dentro del rango de 12 a $30 \mathrm{~Hz}$, se registran en las regiones frontal y central del cerebro y están asociadas con actividades motoras. Los ritmos Beta están sincronizados durante el movimiento real o imágenes motoras. Estas ondas se caracterizan por su distribución simétrica cuando no hay actividad motora. Sin embargo, en caso de movimiento activo, las ondas se atenúan, y sus cambios de distribución son simétricos (Pfurtscheller y Neuper, 2001). Se observa una mayor cantidad de dependencias de nodos del mismo intervalo en esta frecuencia.

A partir de los valores de frecuencia y amplitud obtenidos de la $\mathrm{BN}$ y presentados en la Tabla 1, se construyó el modelo que se muestra en la Figura 1, el cual muestra los estados más probables de voltaje para cada valor de frecuencia. 


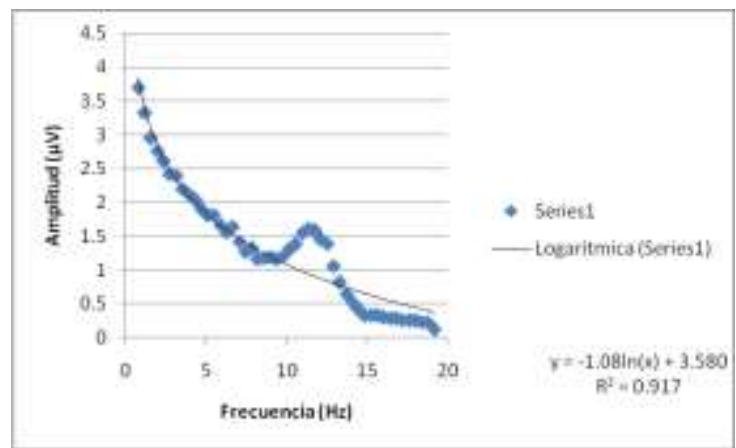

Fig. 1. ---Modelo de BN de las frecuencias para un conjunto de datos EEG.

A partir del modelo BN, se obtuvieron las inferencias. Cada nodo interconectado en la red, presenta un valor de probabilidad condicional el cual cambia en forma encadenada al cambiar los valores de voltaje de cualquier nodo de dicha red. Los valores de voltaje más probables para cada frecuencia, y sus probabilidades condicionales se muestran en la Tabla 1.

El objetivo de la clasificación en un sistema de BCI es el reconocimiento de las intenciones de un usuario en la base de un vector de cierta característica que describe la actividad cerebral, proporcionado por el paso de dicha característica. Cualquiera de los algoritmos de regresión o clasificación se pueden utilizar para lograr este objetivo, pero el uso de algoritmos de clasificación es actualmente el método más popular. Los algoritmos de regresión emplean las características extraídas de las señales de EEG como variables independientes para predecir las intenciones del usuario. En contraste, los algoritmos de clasificación utilizan las características extraídas como variables independientes para definir los límites entre los diferentes objetivos en función del espacio.

\section{Conclusión}

Utilizando un modelo de BN es posible observar y cuantificar las relaciones entre las frecuencias que se expresan en las señales del cerebro, a partir de sus valores de voltaje obtenidos mediante un EEG. El modelo BN muestra que las frecuencias del cerebro dependen unas de otras. Cada nodo interconectado en la red, presenta un valor de probabilidad condicional el cual cambia en forma encadenada al cambiar los valores de voltaje de cualquier nodo de dicha red. En la banda de frecuencias Delta se observan pocas dependencias entre nodos, en las frecuencias Teta, se observa una mayor cantidad de dependencias de nodos del mismo intervalo de frecuencia y con nodos de la frecuencia Beta. En el intervalo Alfa se observan pocas dependencias entre nodos y ninguna con nodos de otras frecuencias, y en la frecuencia Beta se observa una mayor cantidad de dependencias de nodos del mismo intervalo en esta frecuencia. Es necesario continuar trabajos posteriores que determinen las estructuras cerebrales que intervienen en la comunicación de las señales para cada tipo de frecuencias. 


\section{Agradecimientos}

Este trabajo fue parcialmente financiado por el Consejo Nacional de Ciencia y Tecnología (CONACyT) y la Universidad Tecnológica de Corregidora.

\section{Referencias}

1. Anand, B.K.; Chhina, G.S.; Singh, B. Some aspects of electroencephalographic studies in Yogis. Electroencephalogram. Clin. Neurophysiol. 1961, 13, 452-456.

2. Aftanas, L.I.; Golocheikine, S.A. Human anterior and frontal midline theta and lower alpha

Reflect emotionally positive state and internalized attention: High-resolution EEG investigation of meditation. Neurosci. Lett. 2001, 310, 57-60.

3. Baillet, S.; Mosher, J.C.; Leahy, R.M. Electromagnetic brain mapping. IEEE Signal Process. Mag. 2001, 18, 14-30.

4. Black, A.H. The Operant Conditioning of Central Nervous System Electrical Activity. In Psychology of Learning and Motivation, Gordon, H.B., Ed.; Academic Press: New York, NY, USA, 1972; Volume 6, pp. 47-95.

5. Bullier J. Integrated model of visual processing. Brain Res Rev (Review) 2001;36:96-107. Correa M, Bielza C, Paimes-Teixeira J, Alique J R, Comparison of Bayesian networks and artificial neural networks for quality detection in a machining process, Expert Syst Appl, 36 (3) (2009) 7270.

6. Cabeza R, Nyberg L. Imaging cognition. II. An empirical review of 275 PET and fMRI studies. J Cogn Neurosci (Review) 2000;12:1-47.

7. De la torre-Gea G, Soto-Zarazúa G M, Guevara-González R, Rico-García E, Bayesian Networks for defining relations-hips among climate factors, IJPS, 6(18) (2011a) 4412.

8. Fernández, T.; Harmony, T.; Rodríguez, M.; Bernal, J.; Silva, J.; Reyes, A.; Marosi, E. EEG activation patterns during the performance of tasks involving different components of mental calculation. Electroencephalogr. Clin. Neurophysiol. 1995, 94, 175-182.

9. Gámez J A, Mateo J L, Puerta J M, Learning Bayesian networks by hill climbing: efficient methods based on progressive restriction of the neighborhood, Data Min. Knowl. Discov, 22, (2011) 106.

10. Garrote L, Molina M, Mediero L, Probabilistic Forecasts Using Bayesian Networks Calibrated with Deterministic Rainfall-Runoff Models, in Vasiliev et al. edited by Extreme Hydrological Events: New Concepts for Security (Springer) 2007, 173.

11. Guoliang L, Knowledge Discovery with Bayesian Networks, Ph. D. thesis, National University of Singapore, Singapore, 2009.

12. Hruschka E, Hruschka E, Ebecken N F F, Bayesian networks for imputation in classification Problems, J Intell Inform Syst, 29 (2007) 231.

13. Jaime A.P. The functional significance of mu rhythms: Translating "seeing" and "hearing" into "doing". Brain Res. Rev. 2005, 50, 57-68.

14. Klimesch, W. EEG-alpha rhythms and memory processes. Int. J. Psychophysiol. 1997, 26, 319-340.

15. Klimesch, W.; Doppelmayr, M.; Yonelinas, A.; Kroll, N.E.A.; Lazzara, M.; Röhm, D.; Gruber, W. Theta synchronization during episodic retrieval: Neural correlates of conscious awareness. Cogn. Brain. Res. 2001, 12, 33-38. 
16. Kübler, A.; Kotchoubey, B.; Kaiser, J.; Wolpaw, J.R.; Birbaumer, N. Brain-Computer Communication: Unlocking the Locked in; American Psychological Association: Washington, DC, USA, 2001.

17. Leach, W.M., Jr. Fundamentals of low-noise analog circuit design. Proc. IEEE 1994, 82, $1515-1538$.

18. Mediero O L, Probabilistic forecast flood flows Through Bayesian Networks Applied to a Distributed Hydrological Model, Ph. D. thesis, Polytechnic University of Madrid, Madrid, Spain 2007.

19. Mesulam MM. From sensation to cognition (Review). Brain 1998;121:1013-52.

20. Pearl J, Probabilistic reasoning in intelligent systems: networks of plausible inference, edited by Morgan Kaufmann (San Mateo CA, United States) 1988, 64.

21. Pfurtscheller, G.; Neuper, C. Motor imagery and direct brain-computer communication. Proc. IEEE 2001, 89, 1123-1134.

22. Pfurtscheller, G.; Brunner, C.; Schlögl, A.; Lopes da Silva, F.H. Mu rhythm (de)synchronization and EEG single-trial classification of different motor imagery tasks. Neuroimage 2006, 31, 153-159.

23. Reyes P, Bayesian networks for setting genetic algorithm parameters used in problems of geometric constraint satisfaction, Intel. Artificial, 45 (2010) 5.

24. Venables, L.; Fairclough, S. The influence of performance feedback on goal-setting and mental effort regulation. Motiv. Emotion 2009, 33, 63-74.

25. Usakli, A.B. Improvement of EEG signal acquisition: An electrical aspect for state of the art of front end. Comput. Intell. Neurosci. 2010, 2010, 630649.

26. Wang S, Li X, Tang H, Learning Bayesian Networks Structure with Continous Variables, in Li et al., Lecture Notes edited in Computer Science, Heidelberg (Springer-Verlang) 2006, 448.

27. Wolpaw, J.R. Brain-computer interfaces as new brain output pathways. J. Physiol. 2007, $579,613-619$. 